\title{
Reacción anafiláctica secundaria a quiste hidatídico hepático roto*
}

\author{
Drs. JUAN L. MORALES G. ${ }^{1}$, CLAUDIO TAPIA C. ${ }^{1}$, CÉSAR MUÑOZ C. ${ }^{2}$, \\ EDUARDO OTERO V. ${ }^{3}$, RICARDO REBOLLEDO R. ${ }^{1}$ \\ 1 Servicio de Cirugía, Hospital Clínico Herminda Martin, Chillán. \\ 2 Departamento de Cirugía y Traumatología, Universidad de la Frontera, Temuco. \\ 3 Hospital Comunitario, Quirihue. \\ Chile.
}

\begin{abstract}
Anaphylactic reaction secondary to a ruptured liver hydatid cyst. Case report

We report a four years old girl consulting in the emergency room for severe abdominal pain and vomiting secondary to a blunt abdominal trauma during a bicycling accident. A chest and abdomen CAT scan showed ruptured hydatid cysts in the lung and liver and free intraperitoneal fluid. During evolution, the patient developed respiratory distress, bronchial obstruction, a papular exanthema and edema. Due to lack of response to steroids, she was operated excising the ruptured hepatic cyst and performing a peritoneal lavage. The patient had a good postoperative evolution. In a second surgical procedure, the lung cyst was excised. The patient is currently receiving albendazole and is asymptomatic after nine months of follow up.
\end{abstract}

Key words: Hydatidosis, cyst, anaphylaxis, liver.

\section{Resumen}

La hidatidosis es una enfermedad parasitaria, zoonótica y endémica de gran importancia en nuestro país. El quiste hidatídico no complicado suele ser asintomático y es más frecuente encontrarlo en población adulta, siendo infrecuente su diagnóstico en la población pediátrica. Presentamos el caso de una paciente de 4 años de edad derivada al Servicio de Urgencia del Hospital de Chillán por cuadro de abdomen agudo posterior a trauma abdominal con manubrio de bicicleta, cuyo estudio tomográfico reveló la presencia de quiste hidatídico hepático y pulmonar rotos, que evoluciona con reacción anafiláctica secundaria.

Palabras clave: Abdomen agudo, hidatidosis, reacción anafiláctica.

\section{Introducción}

La hidatidosis tiene una prevalencia nacional de 6,3/100.000 habitantes ${ }^{1}$, sin embargo, en algunas regiones, llega a cifras de prevalencia de 48/100.000 habitantes ${ }^{2}$.
El quiste hidatídico (QH) no complicado suele ser asintomático.

Las manifestaciones clínicas del QH pueden estar dadas por las reacciones tóxicas en presencia del parásito, el efecto local y mecánico dado por la localización y vitalidad del quiste, o por sus com-

*Recibido el 9 de junio de 2010 y aceptado para publicación el 12 de diciembre de 2010.

Correspondencia: Dr. Juan L. Morales G.

Avda. Francisco Ramírez s/n. Chillán, Chile. jlmoralesg@ucsc.cl 
plicaciones $^{3}$. Dentro de las complicaciones se ha descrito la obstrucción biliar, la ruptura del quiste y la infección secundaria como las más comunes ${ }^{4}$.

La mayor frecuencia en el diagnóstico de hidatidosis se realiza en la población adulta joven ${ }^{5}$, siendo infrecuente en la población pediátrica.

En una serie pediátrica nacional la edad promedio de presentación fue de 8 años, siendo el QH hepático la localización más frecuente, un 13\% presentaba quistes hepáticos y pulmonares ${ }^{6}$.

La rotura de un $\mathrm{QH}$ puede ser espontánea o traumática. La incidencia de rotura de un $\mathrm{QH}$ es de $3-17 \%{ }^{7}$. La presentación de anafilaxia secundaria a la rotura de un $\mathrm{QH}$ es infrecuente y oscila entre $1-7,5 \%$ de los casos ${ }^{8}$.

Presentamos el caso clínico de una paciente pediátrica que cursó con reacción anafiláctica secundaria a rotura de $\mathrm{QH}$ hepático hacia la cavidad abdominal tratado en el Hospital Clínico Herminda Martín de Chillán.

\section{Caso clínico}

Paciente de género femenino de 4 años de edad, sin antecedentes mórbidos, previamente asintomática, es derivada a la Unidad de Emergencia del Hospital de Chillán por cuadro de 2 horas de evolución caracterizado por compromiso del estado general, dolor abdominal agudo y vómitos, tras sufrir contusión abdominal con el manubrio de una bicicleta.

$\mathrm{Al}$ ingreso se constata presión arterial de 85/50 $\mathrm{mmHg}$, temperatura axilar $38,9^{\circ} \mathrm{C}$ y frecuencia cardíaca 137/minuto. Al examen físico abdominal destacan ruidos hidroaéreos disminuidos, sensibilidad a la palpación, resistencia muscular involuntaria

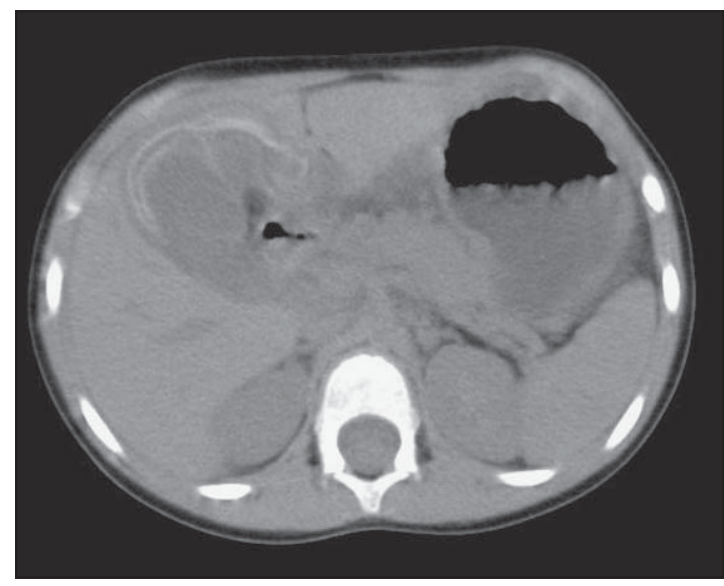

Figura 1. Corte coronal CT Scan Abdominal que muestra quiste hidatídico que compromete gran parte del hígado. y signo de Blumberg. Se decide hospitalizar a la paciente con diagnóstico de trauma abdominal contuso complicado. Se realizan exámenes de laboratorio donde destaca Proteína C Reactiva 51,4 mg/L, hematocrito 34,6\%, hemoglobina 12,4 g/dl, leucocitos $10.000 / \mathrm{mm}^{3}$.

Ante los hallazgos clínicos y de laboratorio se decide realizar tomografía axial computada torácica y abdominal que muestra imagen quística pulmonar derecha con nivel hidroaéreo en su interior, compatible con $\mathrm{QH}$ pulmonar roto estable, e imagen quística en lóbulo hepático derecho con nivel hidroaéreo en su interior y líquido libre intraperitoneal, compatible con QH hepático roto a la cavidad peritoneal (Figuras 1 y 2).

La paciente evoluciona con dificultad respiratoria aguda, obstrucción bronquial, exantema maculopapular generalizado y edema angioneurótico, que se manejaron inicialmente con clorfenamina e hidrocortisona, sin respuesta clínica. La paciente acentuó la sintomatología antes descrita, con mayor compromiso del estado general e inestabilidad hemodinámica, por lo que se decide realizar cirugía de urgencia.

Se realiza laparotomía media supraumbilical que evidencia líquido libre abundante en la cavidad peritoneal y quiste hidatídico multivesicular roto en segmento V hepático. Se procede a cirugía radical (periquistectomía total), colecistectomía incidental y aseo profuso de la cavidad peritoneal con solución fisiológica, sin complicaciones intraoperatorias (Figuras 3 y 4). La paciente se traslada a Unidad de Cuidados Intensivos Pediátricos donde evoluciona

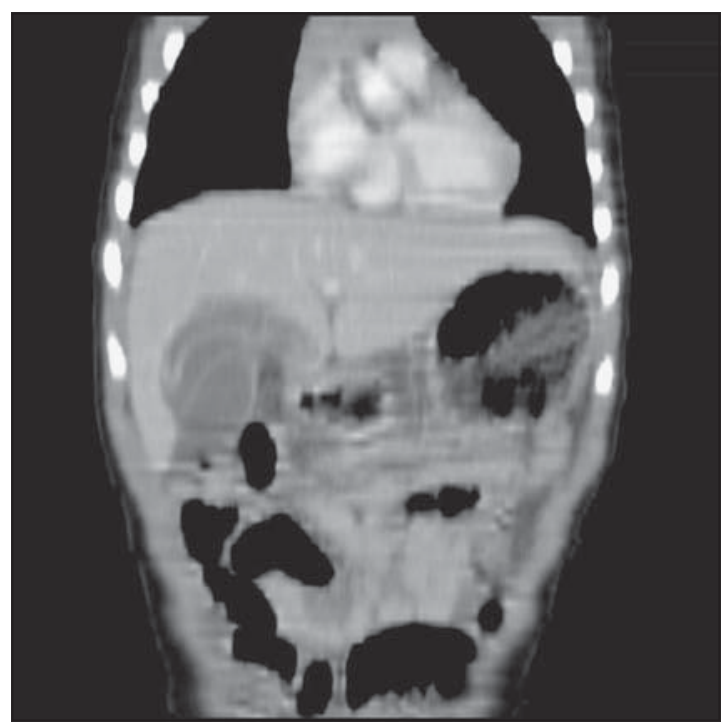

Figura 2. Corte sagital CT Scan Abdominal que muestra compromiso hepático del quiste. 


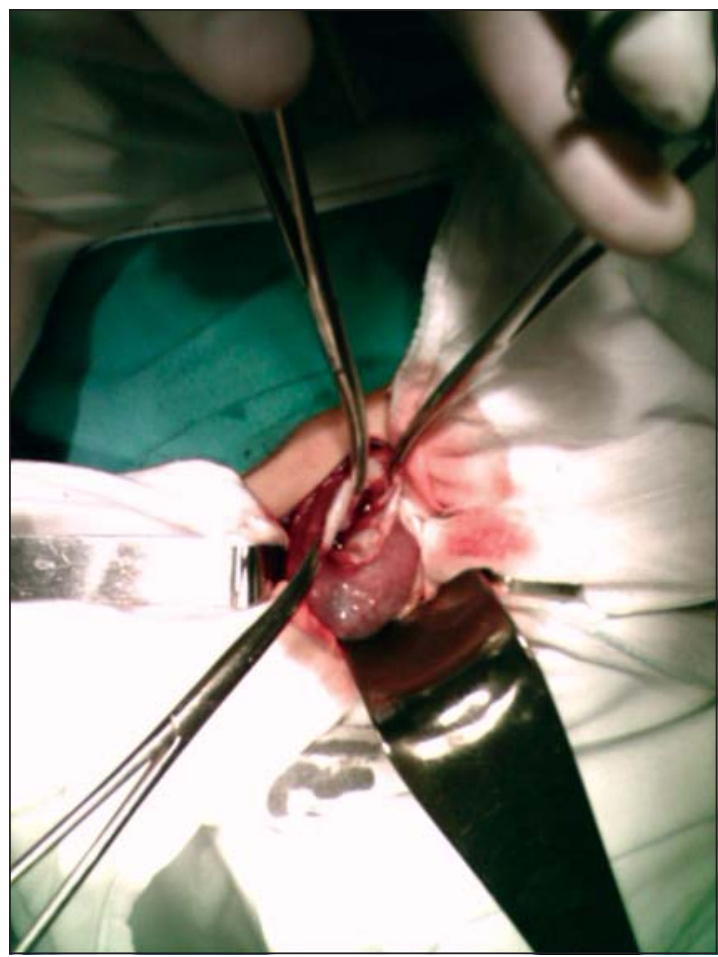

Figura 3. Acceso a quiste hidatídico mediante laparatomía media abdominal.

satisfactoriamente y se inicia tratamiento coadyuvante con albendazol (15 mg/kg/día).

Ante la buena evolución de la paciente se realiza en forma electiva cirugía torácica, donde por toracotomía extramuscular (sin disección muscular) se efectúa quistectomía parcial, sin complicaciones.

La paciente ha completado tres ciclos de 1 mes de terapia coadyuvante con albendazol y no presenta evidencias de recidiva de la enfermedad en los controles tras 9 meses de seguimiento.

\section{Discusión}

La hidatidosis es una enfermedad endémica y un importante problema de salud pública en países de la cuenca Mediterránea, Medio Oriente y Sudaméri$\mathrm{ca}^{9}$. En Chile, las tasas globales de infección varían entre 51-66/100.000 habitantes en la zona norte y $305-657 / 100.000$ en la zona sur, con una mayor prevalencia en las zonas rurales ${ }^{10}$.

El diagnóstico de hidatidosis hepática no complicada está basado en la sospecha clínica y la información epidemiológica, pero generalmente, está dificultado por lo variado del cuadro clínico. Dolor en el cuadrante superior derecho o epigastrio,

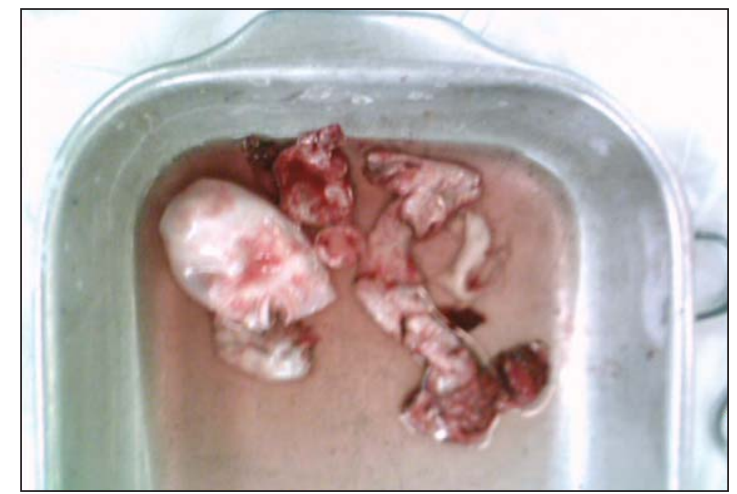

Figura 4. Piezas quirúrgicas extraídas.

hepatomegalia y masa palpable son los síntomas y signos más comunes ${ }^{11}$. También pueden estar presentes síntomas inespecíficos tales como fatiga, fiebre, náuseas y dispepsia ${ }^{12}$.

Pacientes con hidatidosis hepática complicada pueden presentar fiebre, ictericia o anafilaxia, dependiendo de la complicación ${ }^{12}$.

Frecuentemente suelen permanecer asintomáticos por muchos años debido al lento crecimiento de los quistes ${ }^{13}$. Con el tiempo se vuelven más grandes y el peligro de ruptura aumenta. En consecuencia, en un quiste grande, un pequeño trauma puede ser suficiente para romper el quiste, con resultados potencialmente desastrosos ${ }^{9}$. Se ha descrito el desarrollo de shock anafiláctico secundario a un trauma abdominal debido al drenaje del contenido quístico dentro de un vaso sanguíneo periquístico ${ }^{14}$.

El análisis de la literatura sugiere que la presencia de hidatidosis en la población pediátrica es infrecuente, siendo de menor ocurrencia aún, la presentación de este cuadro como una reacción de tipo anafiláctica secundaria a trauma o contusión abdominal. Por ello consideramos de vital importancia incluir dentro del diagnóstico diferencial de todo paciente, adulto o pediátrico, que debute con dolor abdominal agudo, luego del antecedente de un trauma abdominal, la posibilidad de encontrarnos frente a un cuadro de hidatidosis complicada, especialmente en aquellas zonas endémicas, dentro de las cuales se encuentra nuestro país, que cuentan con importantes sectores rurales como es el caso de la provincia de Ñuble.

\section{Referencias}

1. Sapunar J. Hidatidosis en parasitología clínica. En: Atías A, Neghme A (eds). Parasitología Clínica. 2a ed. Publicaciones Técnicas Mediterráneo 1984:297-311. 
2. Manterola C, Ascencio L, Bahamondes J, Barroso M. Hidatidosis hepática. Estudio descriptivo de algunos aspectos clínicos y terapéuticos. Rev Chil Cir. 1997;49:52-9.

3. Sayek I, Onat D. Diagnosis and Treatment of Uncomplicated Hydatid Cyst of the Liver. World J Surg. 2001;25:21-7.

4. Tsaroucha A, Polychronidis A, Lyrantzopoulus N, Pitiakoudis M, Karayiannakis A, Manolas K, et al. Hydatid disease of the abdomen and other locations. World J Surg. 2005;29:1161-5.

5. Aliaga F, Oberg C. Epidemiología de la hidatidosis humana en la IX Región de la Araucanía, Chile. 19911998. Bol Chil Parasitol. 2000;55:54-8.

6. Noemí I, Viovy A, Zamorano R, Blanco A, Revello D, Vojkovic M, y cols. Hidatidosis en la infancia: Albendazol en su tratamiento médico y quirúrgico. Rev Chil Infect. 2003;20:229-34.

7. Kumar R, Reddy S, Thulkar S. Intrabiliary rupture of hydatid cyst: diagnosis with MRI and hepatobiliary isotope study. Br J Radiol. 2002;75:271-4.

8. Ruz M, Delgado E, Gutiérrez A, Suárez M. Shock anafiláctico como primera manifestación de enfermedad hidatídica hepática. Rev Esp Enferm Dig. 1991;98:70-1.

9. Kantarci M, Onbas O, Alper F, Celebi Y, Yigiter M, Okur A. Anaphylaxis due to a rupture of hydatid cyst: Imaging findings of a 10-year-old boy. Emergency Radiology 2003;10:49-50.

10. Schenone H, Contreras M, Salinas P, Sandoval L, Saavedra T, Rojas A. Hidatidosis humana en Chile. Seroprevalencia y estimación del número de personas infectadas. Bol Chil Parasitol. [online]. 1999;54:3-4 [citado 2009-10-07], pp. 70-73. Disponible en: http://www. scielo.cl/scielo.php?script=sci_arttext\&pid=S036594021999000300006\&lng=es\&nrm=iso

11. Sayek I, Yalin R, Sanac Y. Surgical treatment of hydatid disease of the liver. Arch Surg. 1980;115:847-50.

12. Sayek I, Tirnaksiz M, Dogan R. Cystic Hydatid Disease: Current Trends in Diagnosis and Management. Surg Today 2004;34:987-96.

13. Celebi F, Balik A, Salman A, Ören D. Hydatid disease in childhood. Pediatr Surg Int. 2002;18:417-9.

14. Eyal I, Zveibil F, Stamler B. Anaphylactic shock due to rupture of a hepatic hydatid cyst into a pericystic blood vessel following blunt abdominal trauma. J Pediatr Surg. 1991;26:217-8. 